\title{
Factors associated with the development of septic shock in patients with candidemia: a post hoc analysis from two prospective cohorts
}

\author{
Matteo Bassetti ${ }^{1,23^{*}}$ D, Antonio Vena ${ }^{1,2,3}$, Marco Meroi ${ }^{1}$, Celia Cardozo ${ }^{4}$, Guillermo Cuervo ${ }^{5}$, \\ Daniele Roberto Giacobbe ${ }^{2,3}$, Miguel Salavert ${ }^{6}$, Paloma Merino ${ }^{7}$, Francesca Gioia ${ }^{8}$, Mario Fernández-Ruiz ${ }^{9}$, \\ Luis Eduardo López-Cortés ${ }^{10}$, Benito Almirante ${ }^{11}$, Laura Escolà-Vergé ${ }^{11}$, Miguel Montejo ${ }^{12}$, Manuela Aguilar-Guisado ${ }^{13}$, \\ Pedro Puerta-Alcalde", Mariona Tasias ${ }^{6}$, Alba Ruiz-Gaitán ${ }^{6}$, Fernando González ${ }^{7}$, Mireia Puig-Asensio ${ }^{11}$, \\ Francesc Marco ${ }^{4}$, Javier Pemán ${ }^{6}$, Jesus Fortún ${ }^{8}$, Jose Maria Aguado ${ }^{9}$, Alejandro Soriano ${ }^{4}$, Jordi Carratalá, \\ Carolina Garcia-Vidal ${ }^{4}$, Maricela Valerio ${ }^{14,15}$, Assunta Sartor ${ }^{16}$, Emilio Bouza ${ }^{14,15,17,18}$ and Patricia Muñoz ${ }^{14,15,17,18}$
}

\begin{abstract}
Background: Almost one third of the patients with candidemia develop septic shock. The understanding why some patients do and others do not develop septic shock is very limited. The objective of this study was to identify variables associated with septic shock development in a large population of patients with candidemia.

Methods: A post hoc analysis was performed on two prospective, multicenter cohort of patients with candidemia from 12 hospitals in Spain and Italy. All episodes occurring from September 2016 to February 2018 were analyzed to assess variables associated with septic shock development defined according to The Third International Consensus Definition for Sepsis and Septic Shock (Sepsis-3).

Results: Of 317 candidemic patients, 99 (31.2\%) presented septic shock attributable to candidemia. Multivariate logistic regression analysis identifies the following factors associated with septic shock development: age $>50$ years (OR 2.57, $95 \% \mathrm{Cl} 1.03-6.41, p=0.04)$, abdominal source of the infection (OR 2.18, 95\% Cl 1.04-4.55, $p=0.04)$, and admission to a general ward at the time of candidemia onset (OR $0.21,95 \% \mathrm{Cl}, 0.12-0.44, p=0.001)$. Septic shock development was independently associated with a greater risk of 30-day mortality (OR 2.14, 95\% Cl 1.08-4.24, $p=0.02)$.
\end{abstract}

Conclusions: Age and abdominal source of the infection are the most important factors significantly associated with the development of septic shock in patients with candidemia. Our findings suggest that host factors and source of the infection may be more important for development of septic shock than intrinsic virulence factors of organisms.

Keywords: Candidemia, Septic shock, Intra-abdominal candidiasis, Risk factors

\footnotetext{
* Correspondence: matteo.bassetti@unige.it

'Infectious Diseases Clinic, Department of Medicine, University of Udine and

Azienda Sanitaria Universitaria Integrata, Piazzale Santa Maria della

Misericordia 15, 33010 Udine, Italy

${ }^{2}$ Department of Health Sciences, University of Genoa, Genoa, Italy

Full list of author information is available at the end of the article
}

(C) The Author(s). 2020 Open Access This article is licensed under a Creative Commons Attribution 4.0 International License, which permits use, sharing, adaptation, distribution and reproduction in any medium or format, as long as you give appropriate credit to the original author(s) and the source, provide a link to the Creative Commons licence, and indicate if changes were made. The images or other third party material in this article are included in the article's Creative Commons licence, unless indicated otherwise in a credit line to the material. If material is not included in the article's Creative Commons licence and your intended use is not permitted by statutory regulation or exceeds the permitted use, you will need to obtain permission directly from the copyright holder. To view a copy of this licence, visit http://creativecommons.org/licenses/by/4.0/ The Creative Commons Public Domain Dedication waiver (http://creativecommons.org/publicdomain/zero/1.0/) applies to the data made available in this article, unless otherwise stated in a credit line to the data. 


\section{Introduction}

Candida species has been reported as the most common cause of fungal disease in septic shock, affecting $8-10 \%$ of these patients [1-3]. Moreover, among patients developing septic shock, candidemia is actually considered the clinical condition with the highest attributable mortality, ranging from 54 to $66 \%$ [4-6]. The survival of these patients is strictly related to a timely control of the source and appropriate antifungal treatment $[4,5]$.

While candidemia has long been recognized as a trigger for septic shock development, our understanding of why patients with candidemia develop or not septic shock has not been well addressed [7]. Literature on the topic is very limited and difficult to interpret due to the low number of patients included and the inconclusive results reported [7].

Therefore, the aim of our study was to assess factors associated with the development of septic shock in the context of two large multicenter studies of patients with candidemia.

\section{Materials and methods}

\section{Study design, setting, and population}

In the present report, we included data coming from two separate studies. First is a prospective cohort assembled within a quasi-experimental study aimed at assessing the impact of an evidence-based intervention bundle to improve the clinical management of candidemia (the CANDI-Bundle Study) that was conducted from September 2016 to February 2018 in 11 hospitals from Spain. Second is a prospective observational study on candidemia that was conducted during the same period at the University Hospital Santa Maria della Misericordia in Udine, Italy. The full results from both cohorts are currently undergoing detailed analysis.

In order to ensure comparability, the same inclusion and exclusion criteria, study definitions, patient followup, and data collection procedures were applied in both studies. However, for this post hoc analysis, we excluded patients aged $<18$ years old and those who had mixed infection (concomitant blood culture positive for a bacterial pathogen).

The study was approved by the local institutional review boards, and written patient consent was not required because of observational nature of this study.

\section{Clinical data and definitions}

Data were collected from patients' hospital charts and the laboratory database, which contains complete profiles for all candidemic patients. The main outcome measured was development of septic shock attributable to candidemia. Septic shock patients and non-septic shock subgroups were compared to identify variables associated with septic shock development.
The following variables were explored as possibly associated with septic shock development:

i. For patient variables, we considered age, sex, hospital ward stay at the time of infection, Charlson comorbidity index [8]), underlying diseases, immunosuppressive therapy, history of previous abdominal surgery (30 days preceding candidemia onset), presence of central venous catheter (CVC) at the time of candidemia, total parenteral nutrition, and prior exposure to antimicrobial or antifungal therapy (within previous 30 days).

ii. For infection variables, we considered source of infection, pathogen species, and antifungal resistance.

All patients were followed until discharge or death and were assessed for survival on day 30.

\section{Definitions}

An episode of candidemia was defined as at least one peripheral blood culture positive for Candida spp. Candidemia onset was defined as the date of collection of the first blood culture yielding the study isolate.

Septic shock was determined according to The Third International Consensus Definition for Sepsis and Septic Shock (Sepsis-3) [9]. All clinical and laboratory data defining septic shock were assessed within $24 \mathrm{~h}$ of the first positive blood culture yielding Candida spp. Patients were classified as having septic shock attributable to Candida infection if vasopressors were required to maintain a mean arterial pressure of $\geq 65 \mathrm{mmHg}$ and serum lactate level greater than $2 \mathrm{mmol} / \mathrm{L}$ [9]. Patients who required vasopressors $\geq 24 \mathrm{~h}$ prior to the collection of a blood culture subsequently positive for Candida species were included in the non-septic shock group.

As for the source of infection, CVC-related candidemia was defined according to current guidelines $[10,11]$. The urinary tract was considered to be the portal of entry in patients with urological predisposing conditions (i.e., manipulation or obstruction of the urinary tract) and evidence of urinary tract infection caused by the same Candida spp. [12]. The abdomen was considered to be the origin of the candidemia when a patient had evidence of abdominal infection and (i) a positive culture from the intra-abdominal space was obtained during surgery or by needle aspiration or (ii) no other apparent sources of candidemia were detected [13-15]. When a source of candidemia could not be identified, candidemia was defined as "primary."

Initial antifungal therapy was considered as adequate if at least one active drug according to in vitro susceptibility results was initiated at an appropriate dosage [10] within the first $24 \mathrm{~h}$ after blood culture was obtained. 
Source control included CVC withdrawal and invasive procedures to resolve urinary tract obstruction and intra-abdominal abscess drainage, depending on the source of candidemia. Source control was considered adequate when performed within $24 \mathrm{~h}$ from candidemia onset.

Neutropenia was defined as an absolute neutrophil count $<500$ cells $/ \mathrm{mm}^{3}$ at the onset of candidemia.

\section{Microbiological studies}

Candida specie identification and in vitro antifungal activity were assessed at participating hospitals using local routine methods. Currently, two sets of two blood samples were collected from patients with a suspected bloodstream infection. The blood samples were processed using a BACTEC 9240 system (Becton-Dickinson Microbiology Systems, Franklin Lakes, NJ, USA) or BACTE 860 system or BacTAlert (BioMérieux SA, Marcy L'Etoile, France) with an incubation period of 5 days. If yeast cells were observed after microscopic examination of a Gram stain, blood bottles were subcultured into Sabouraud agar plates (BD BBL StrackerTM PlatesTM, Heidelberg, Germany) and chromogenic media (ChromAgar BioMerieux SA, Paris, France). In vitro antifungal activity was studied by a commercial microdilution method (YeastOne ${ }^{\oplus}$ Sensitre ${ }^{\oplus}$, TREK Diagnostic Systems Ltd., East Grinstead, UK) or E test (BioMérieux SA, Paris, France), in accordance with the manufacturer's instructions.

\section{Statistical analysis}

Categorical variables are expressed as absolute numbers and relative frequencies. Quantitative variables are presented as means and standard deviation (SD) if normally distributed or as median and interquartile range (IQR) if non-normally distributed. We compared continuous variables between patients with or without septic shock using the Student $t$ test for normally distributed variables and the Mann-Whitney $U$ test for non-normally distributed variables. Categorical variables were evaluated by using the chi-square or the two-tailed Fisher exact test.

Variables associated with septic shock due to candidemia in univariate analysis $(p \leq 0.20)$ were included in a logistic regression analysis, and a backward stepwise approach was used to identify those independently associated with septic shock development $(p<0.05)$. All statistical analyses were performed by using SPSS Statistics for MAC, Version 22.0 (SPSS Inc., Chicago, IL, USA).

\section{Results}

A total of 317 episodes of candidemia were included in the study. Overall, 99 of the 317 patients (31.2\%) fulfilled the criteria for septic shock within the first $24 \mathrm{~h}$ after blood culture was drawn, and $34.3 \%$ of them died in 30 days.

\section{Clinical characteristics of study population}

Demographics and baseline clinical features of study population are shown in Table 1. Mean age was 65.4 years, and 184 patients were men (58.0\%). Overall, 63 out of 317 (19.9\%) episodes of candidemia occurred in intensive care unit (ICU), and 254 (80.1\%) occurred in patients hospitalized in general wards, including 150 (47.3\%) in internal medicine and $104(32.8 \%)$ in surgical wards.

The most common underlying condition was solid tumor (35.9\%) followed by cardiovascular disease (32.2\%) and diabetes mellitus (28.4\%). Almost all patients had recently received antibiotic therapy (within the previous 1 month). A CVC was in place in 225 out of 317 patients $(71.0 \%)$, with 158 of them receiving total parenteral nutrition at the time of candidemia onset. The most prevalent source of infection was the CVC (44.5\%) followed by the abdomen that was detected in 39 patients $(12.3 \%)$. Source of candidemia remained unknown in 98 (30.9\%) patients.

As for species, C. albicans was the most frequent one (42.3\%) followed by C. glabrata (18.3\%), C. parapsilosis (17.4\%), and C. tropicalis (9.1\%).

\section{Variables associated with septic shock attributable to candidemia}

Patients with septic shock attributable to candidemia (99 patients) and without septic shock (218 patients) were compared (Table 1). There were no significant differences regarding sex, Charlson comorbidity index, underlying disease, and risk factors for candidemia and Candida species. However, at univariate analysis, factors associated with development of septic shock were as follows: age older than 50 years $(92.9 \%$ vs $83.9 \%$; $p=0.03)$, hospitalization in ICU at the time of candidemia $(37.4 \%$ vs $11.9 \%, p=0.001$ ), and an abdominal source of the infection $(20.2 \%$ vs $8.7 \%$; $p=0.006)$. A multivariate analysis (Table 2) showed that older age (OR 2.57, 95\% CI 1.036.41, $p=0.04$ ) and an abdominal source of the infection (OR 2.18, 95\% CI 1.04-4.55, $p=0.04$ ) were independently associated with the development of septic shock in candidemic patients. By contrast, being admitted to a general ward at the time of candidemia onset was considered as a protective factor (OR 0.21, 95\% CI $0.12-$ $0.44, p=0.001$ ).

Comparison between patients with and without intraabdominal origin of the infection is reported in Supplementary material 1. As for infection management, echinocandin was the most frequent initial antifungal agent, prescribed in $57.7 \%$ of cases, followed by azoles in $35.3 \%$ of patients. Although rates of initial echinocandin 
Table 1 Comparison of the main demographic and clinical characteristics of study population

\begin{tabular}{|c|c|c|c|c|}
\hline Variable & All episodes, $N=317(\%)$ & Non-septic shock, $N=218(\%)$ & Septic shock, $N=99(\%)$ & $p$ value \\
\hline \multicolumn{5}{|l|}{ Demographics } \\
\hline Sex, male & $184(58.0)$ & $141(64.7)$ & $43(43.4)$ & 0.17 \\
\hline Mean age $( \pm S D)$, years & $65.4 \pm 17.0$ & $65.2 \pm 18.6$ & $66.9 \pm 15.2$ & 0.77 \\
\hline Age $\geq 50$ years & $275(86.4)$ & $183(83.9)$ & $92(92.9)$ & 0.03 \\
\hline Median hospital stay until Candida BSI (IQR), days & $16(7-31)$ & $17(6-32)$ & $14(8-28)$ & 0.18 \\
\hline \multicolumn{5}{|l|}{ Hospital ward stay at the time of candidemia onset } \\
\hline General ward & $254(80.1)$ & $192(88.1)$ & $62(62.6)$ & 0.001 \\
\hline Intensive care unit & $63(19.9)$ & $26(11.9)$ & $37(37.4)$ & \\
\hline Charlson comorbidity index & $3.4 \pm 2.7$ & $3.4 \pm 2.6$ & $3.3 \pm 2.8$ & 0.71 \\
\hline \multicolumn{5}{|l|}{ Underlying condition } \\
\hline Solid tumor & $114(35.9)$ & $77(35.3)$ & $37(37.4)$ & 0.80 \\
\hline Cardiovascular disease & $102(32.2)$ & $69(31.7)$ & $33(33.3)$ & 0.79 \\
\hline Diabetes mellitus & $90(28.4)$ & $57(26.1)$ & $33(33.3)$ & 0.22 \\
\hline Chronic Lung disease & $51(16.1)$ & $35(16.1)$ & $16(16.2)$ & 1 \\
\hline Chronic kidney failure & $50(15.8)$ & $32(14.7)$ & $18(18.2)$ & 0.50 \\
\hline Chronic liver disease & $41(12.9)$ & $27(12.4)$ & $14(14.1)$ & 0.74 \\
\hline Solid organ transplantation & $27(8.5)$ & $19(8.7)$ & $8(8.1)$ & 1 \\
\hline Hematological malignancy & $20(6.3)$ & $16(7.3)$ & $4(4.0)$ & 0.32 \\
\hline Hematopoietic stem cell transplantation & $9(2.8)$ & $8(3.7)$ & $1(1.0)$ & 0.28 \\
\hline \multicolumn{5}{|l|}{ Risk factors for candidemia } \\
\hline Previous antibiotic therapy & $297(93.6)$ & $202(92.7)$ & $95(96.0)$ & 0.32 \\
\hline Central venous catheter & $225(71.0)$ & $150(68.8)$ & $75(75.8)$ & 0.23 \\
\hline TPN during candidemia & $158(49.8)$ & $101(46.3)$ & $57(57.6)$ & 0.07 \\
\hline Previous corticosteroid therapy & $72(22.7)$ & $51(23.4)$ & $21(22.1)$ & 0.77 \\
\hline Abdominal surgery & $85(26.8)$ & $52(23.9)$ & $33(33.3)$ & 0.10 \\
\hline Immunosuppressive therapy & $47(14.8)$ & $36(16.5)$ & $11(11.1)$ & 0.23 \\
\hline Neutropenia & $10(3.2)$ & $9(4.1)$ & $1(1.0)$ & 0.18 \\
\hline Previous antifungal treatment & $49(15.4)$ & $37(17.0)$ & $12(12.1)$ & 0.32 \\
\hline \multicolumn{5}{|l|}{ Source of infection } \\
\hline Central venous catheter & $141(44.5)$ & $101(46.3)$ & $40(40.4)$ & 0.33 \\
\hline Primary & $98(30.9)$ & $73(33.5)$ & $25(25.3)$ & 0.15 \\
\hline Abdomen & $39(12.3)$ & $19(8.7)$ & $20(20.2)$ & 0.006 \\
\hline Urinary tract & $23(7.3)$ & $18(8.3)$ & $5(5.1)$ & 0.36 \\
\hline Infective endocarditis & $3(0.9)$ & $1(0.5)$ & $2(2.0)$ & 0.23 \\
\hline Other & $13(4.1)$ & $6(2.8)$ & $7(7.1)$ & 0.12 \\
\hline \multicolumn{5}{|l|}{ Candida species } \\
\hline C. albicans & $134(42.3)$ & $91(41.7)$ & $43(43.4)$ & 0.80 \\
\hline C. glabrata & $58(18.3)$ & $38(17.4)$ & $20(20.2)$ & 0.63 \\
\hline C. parapsilosis & $55(17.4)$ & $40(18.3)$ & $15(15.2)$ & 0.52 \\
\hline C. tropicalis & $29(9.1)$ & $22(10.1)$ & $7(7.1)$ & 0.52 \\
\hline C. krusei & $6(1.9)$ & $4(1.8)$ & $2(2.0)$ & 1 \\
\hline C. Iusitaniae & $4(1.3)$ & $2(0.9)$ & $2(2.0)$ & 0.59 \\
\hline C. auris & $27(8.5)$ & $18(8.3)$ & $9(9.1)$ & 0.86 \\
\hline Other & $13(4.1)$ & $11(5.0)$ & $2(2.0)$ & 0.35 \\
\hline
\end{tabular}


Table 1 Comparison of the main demographic and clinical characteristics of study population (Continued)

\begin{tabular}{|c|c|c|c|c|}
\hline Variable & All episodes, $N=317(\%)$ & Non-septic shock, N=218 (\%) & Septic shock, $N=99(\%)$ & $p$ value \\
\hline \multicolumn{5}{|l|}{ Resistant strains } \\
\hline Fluconazole & $45(14.1)$ & 29 (13.3) & $16(16.2)$ & 0.73 \\
\hline Echinocandins & $3(0.9)$ & $2(0.9)$ & $1(1.0)$ & 1 \\
\hline
\end{tabular}

$B S I$ bloodstream infection, IQR interquartile range, $S D$ standard deviation, $T P N$ total parenteral nutrition

therapy $(65.3 \%$ vs $60.9 \%)$ or initial azole therapy $(32.7 \%$ vs $39.1 \%)$ were comparable, patients with an intra-abdominal origin received an adequate initial antifungal treatment more often (38.1 vs $61.5, p=0.008)$. No differences regarding the rates of adequate source control (43.4\% vs $40.5 \%$, $p=0.86)$ were observed between groups.

\section{Prognostic factors for 30-day mortality}

We compared candidemic patients who died at 30 days (70 patients) with those who survived (247 patients). Septic shock attributable to candidemia was present in $48.6 \%$ of patients who died (34/70) in comparison to $26.3 \% \quad(65 / 247$ patients $)$ who survived $(p=0.001)$ (Table 3). The Kaplan-Meier curves in Fig. 1 demonstrate that patients with septic shock attributable to candidemia had a significantly greater likelihood of dying compared to patients not developing septic shock at candidemia onset.

Variables examined as risk factors for 30-day mortality are presented in Table 3. Multivariate logistic regression analysis demonstrated that a higher Charlson comorbidity index and septic shock attributable to candidemia and requiring hemodialysis after candidemia onset were independently associated with greater 30-day mortality, while CVC-related candidemia was independently associated with a lower risk of 30-day mortality (Table 3).

\section{Discussion}

To the best of our knowledge, the current multicenter study is the largest one to identify variables associated with development of septic shock among patients with Candida bloodstream infection. We found a significant rate of septic shock among patients with candidemia (30\%), and we also demonstrated that age more than 50 years old and an intra-abdominal origin of the infection are the most important independent factors associated with septic shock development.
The incidence of septic shock is only collaterally mentioned in reports on candidemia in the general populations and it ranges from 12.9 to $49.0 \%$ in recent studies [16-18]. However, only three series have specifically focused on the incidence of septic shock among candidemic patients. Patel et al. [19] in a single center retrospective study that ended in 2007 documented septic shock in $23 \%$ of patients with candidemia. The prevalence was higher in two cohorts of critically ill patients with candidemia in which septic shock developed in $35 \%$ of them [7, 20]. Ours is the first study to analyze the prevalence of septic shock attributable to candidemia, in the light of the new Sepsis-3 definition [9]. With these new criteria, we found that septic shock occurred in almost one third of unselected candidemic patients.

We also found a significant difference in term of prevalence between ICU patients and those admitted to internal medicine and surgical ward, with a nearly two times greater proportion of septic shock in ICU patients. Although such difference was not unexpected, an underestimation of the number of cases of septic shock outside ICU should not be excluded. Regarding this aspect, new Sepsis-3 definitions showed low sensitivity in non-critically ill patients and performed poorly as a screening tool for early identification of sepsis outside ICU [21-23] Moreover, many patients admitted to internal medicine and surgical ward are frequently affected by multiple pre-existing underlying diseases [24-28]. In such circumstance, worsening of clinical conditions can be often misled to the progression of the underlying disease rather than to infection, as suggested by the low number of patients who were admitted to ICU after candidemia onset in our study $(\sim 7 \%)$.

Factors predisposing candidemic patients to develop septic shock have been reported in just one retrospective study including only 15 patients with septic shock [7].

Table 2 Risk factors for development of septic shock at candidemia onset. Multivariate analysis

\begin{tabular}{llll}
\hline Variable & Odds ratio & $95 \%$ confidence interval & $p$ value \\
\hline Age $>50$ years & 2.57 & $1.03-6.41$ & $\mathbf{0 . 0 4}$ \\
Intra-abdominal source of the infection & 2.18 & $1.04-4.55$ & $\mathbf{0 . 0 4}$ \\
Male sex & 1.33 & $0.78-2.26$ & 0.29 \\
Charlson comorbidity index & 0.99 & $0.91-1.10$ & 0.92 \\
General ward stay at the time of candidemia onset & 0.21 & $0.12-0.44$ & $\mathbf{0 . 0 0 1}$ \\
\hline
\end{tabular}


Table 3 Risk factors for 30-day mortality (univariate and multivariate analysis)

\begin{tabular}{|c|c|c|c|c|c|c|c|}
\hline Variable & All episodes, $N=317(\%)$ & Alive, $N=247(\%)$ & Died, $N=70$ (\%) & $p$ & OR & $95 \% \mathrm{Cl}$ & $p$ \\
\hline \multicolumn{8}{|l|}{ Demographics } \\
\hline Sex, male & $184(58.0)$ & $154(62.3)$ & $46(61.4)$ & 0.89 & - & - & - \\
\hline Age $\geq 50$ years & $275(86.8)$ & $212(85.8)$ & $63(90.0)$ & 0.43 & - & - & - \\
\hline Mean age $( \pm S D)$, years & $65.4 \pm 17.0$ & $64.8 \pm 16.8$ & $67.2 \pm 17.7$ & 0.30 & - & - & - \\
\hline $\begin{array}{l}\text { Median hospital stay until } \\
\text { Candida BSI (IQR), days }\end{array}$ & $16(7-31)$ & $15(6-29)$ & $20(10-34)$ & 0.09 & - & - & \\
\hline \multicolumn{8}{|l|}{ Hospital ward } \\
\hline General ward & $254(80.1)$ & $201(84.1)$ & $47(67.1)$ & 0.003 & 1.9 & $0.93-4.30$ & 0.78 \\
\hline Intensive care unit & 63 (19.9) & $38(15.9)$ & $23(32.9)$ & & & & \\
\hline Charlson comorbidity index & $3.4 \pm 2.7$ & $3.0 \pm 2.4$ & $4.6 \pm 3.1$ & $<0.001$ & 1.28 & $1.13-1.45$ & $<0.001$ \\
\hline \multicolumn{8}{|l|}{ Underlying condition } \\
\hline Solid tumor & $114(35.9)$ & $91(36.8)$ & $23(32.9)$ & 0.57 & - & - & - \\
\hline Cardiovascular disease & $102(32.2)$ & $72(29.1)$ & $30(42.9)$ & 0.04 & - & - & - \\
\hline Diabetes mellitus & $90(28.4)$ & $68(27.5)$ & $22(31.4)$ & 0.55 & - & - & - \\
\hline Chronic lung disease & $51(16.1)$ & $36(14.6)$ & $15(21.4)$ & 0.20 & - & - & - \\
\hline Chronic kidney failure & $50(15.8)$ & $36(14.6)$ & $14(20.0)$ & 0.27 & - & - & - \\
\hline Chronic liver disease & $41(12.9)$ & $26(10.5)$ & $15(21.4)$ & 0.02 & - & - & - \\
\hline Solid organ transplantation & $27(8.5)$ & $22(8.9)$ & $5(7.1)$ & 0.81 & - & - & - \\
\hline Hematological malignancy & $20(6.3)$ & $15(6.1)$ & $5(7.1)$ & 0.78 & - & - & - \\
\hline Hematopoietic stem cell transplantation & $9(2.8)$ & $6(2.4)$ & $3(4.3)$ & 0.41 & - & - & - \\
\hline \multicolumn{8}{|l|}{ Risk factors for candidemia } \\
\hline Previous antibiotic therapy & $297(93.6)$ & $232(93.9)$ & $65(92.9)$ & 0.78 & - & - & - \\
\hline Central venous catheter & $225(71.0)$ & $171(69.2)$ & $54(77.1)$ & 0.23 & - & - & - \\
\hline TPN during candidemia & $158(49.8)$ & $118(47.8)$ & $40(57.1)$ & 0.17 & - & - & - \\
\hline Previous corticosteroid therapy & $72(22.7)$ & $53(21.5)$ & $19(27.1)$ & 0.33 & - & - & - \\
\hline Abdominal surgery & $85(26.8)$ & $65(26.3)$ & $20(28.6)$ & 0.76 & - & - & - \\
\hline Immunosuppressive therapy & $47(14.8)$ & $39(15.8)$ & $8(11.4)$ & 0.45 & - & - & - \\
\hline Neutropenia & $10(3.2)$ & $7(2.8)$ & $3(4.3)$ & 0.46 & - & - & - \\
\hline Previous antifungal treatment & $49(15.4)$ & $37(15.0)$ & $12(17.1)$ & 0.71 & - & - & - \\
\hline \multicolumn{8}{|l|}{ Source of infection } \\
\hline Central venous catheter & $141(44.5)$ & $122(49.9)$ & $19(27.1)$ & 0.001 & 0.42 & $0.20-0.88$ & 0.02 \\
\hline Primary & $98(30.9)$ & $71(28.7)$ & $27(38.6)$ & 0.14 & - & - & \\
\hline Abdomen & $39(12.3)$ & $22(8.9)$ & $17(24.3)$ & 0.001 & 1.89 & $0.76-4.66$ & 0.17 \\
\hline Urinary tract & $23(7.3)$ & $22(8.9)$ & $1(1.4)$ & 0.03 & 0.11 & $0.12-1.02$ & $0.05-$ \\
\hline Infective endocarditis & $3(0.9)$ & $3(1.2)$ & $0(0)$ & 1 & - & - & - \\
\hline Other & $13(4.1)$ & $7(2.8)$ & $6(8.6)$ & 0.05 & - & - & - \\
\hline Septic shock & $99(31.2)$ & $65(26.3)$ & $34(48.6)$ & 0.001 & 2.14 & $1.08-4.24$ & 0.02 \\
\hline Initial adequate antifungal therapy & $130(41.0)$ & $95(38.5)$ & $35(50.0)$ & 0.09 & 1.41 & $0.71-2.81$ & 0.32 \\
\hline Adequate source control of the infection* & 124/288 (43.1) & $94 / 225(41.8)$ & 30/63 (47.6) & 0.47 & 1.68 & $0.84-3.35$ & 0.14 \\
\hline Hemodialysis after candidemia onset & $7(2.2)$ & $2(0.8)$ & $5(7.1)$ & 0.007 & 8.55 & $1.35-53.95$ & 0.02 \\
\hline
\end{tabular}

*The source of the infection was susceptible of control in 288 patients

$B S /$ bloodstream infection, IQR interquartile range, SD standard deviation, TPN total parenteral nutrition 


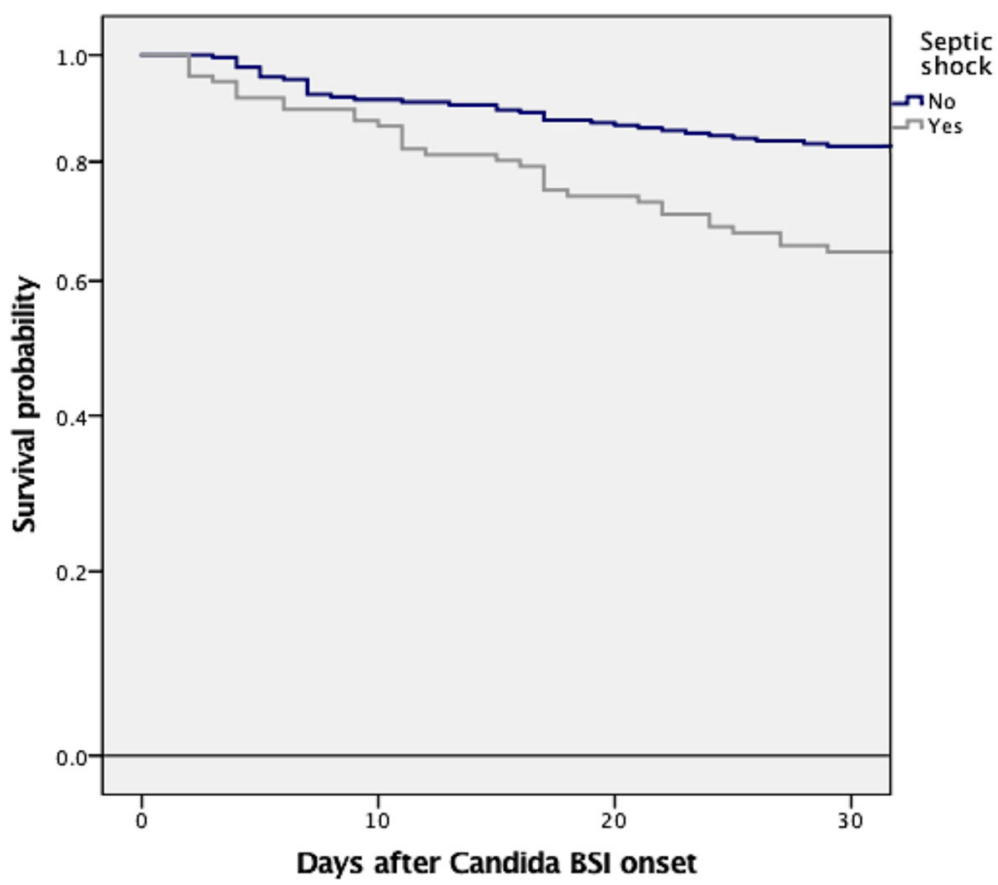

Fig. 1 Thirty-day mortality of candidemic patients according to the development or not of septic shock, log rank $\leq 0.001$

Reflecting this weakness, this study identified no conventional factors associated with septic shock development other than the time spent in ICU before candidemia onset.

Our data suggest that, apart from older age, the only variable associated with septic shock in candidemic patient was an intra-abdominal origin of the infection. This result supports recent experimental observations that intestinal abundance of Candida may be associated with an increased sepsis severity, perhaps through cytokine storm induction and/or decreased macrophage killing activity [29-31]. The fact that patients with other source of the infection (i.e., CVC or urinary tract) received a similar rate of adequate source control of candidemia ( $43.4 \%$ vs $40.5 \%$, $p=0.86$ ) and even a lower rate of adequate antifungal treatment ( $38.1 \%$ vs $61.5 \%, p=0.008)$ is further consistent with this intriguing explanation. Although we do not have clear answer regarding to the best approach for reducing the incidence of septic shock in candidemic patients, we believe that new diagnostic strategies investigating the role of serological biomarkers such as $\beta$-D-glucan or T2MR [32-35] should be applied in order to early identify patients at risk of intra-abdominal candidiasis. Future studies addressing risk factors for developing intra-abdominal candidiasis are also needed to better clarify the best empiric or pre-emptive therapeutic approach.

With regard to Candida species as predisposing factor, Guzman et al. did [36] not find any significant differences [7]. Despite our study including 27 episodes of $C$. auris candidemia (8.5\%) that is typically associated with a high degree of virulence [37], we confirm previous results, thus suggesting that host factors may be more important than intrinsic factors of organisms. Antifungal resistance was not an issue predisposing to septic shock in our study, since only $14.1 \%$ of the patients had fluconazole resistant strains and half of them received adequate definitive antifungal treatment.

Of interest, in the present report, the overall 30-day mortality rate in patients with septic shock attributable to candidemia was $34.3 \%$, which is significantly lower than that observed in two previous studies performed in Europe and St. Louis, where mortality rate remained around $50-60 \%[4,5]$. The difference in septic shock definitions [9], improvements in fungal diagnosis [33, 34], and the widespread implementation of sepsis bundle in recent years [38-40] may explain the lower incidence of mortality observed in this study.

As for risk factors for mortality, our findings are consistent with those recently reported by other investigators [41] who found increased severity of the underlying conditions (higher Charlson comorbidity index), clinical presentation of candidemia (septic shock), and signs of organ dysfunction (hemodialysis after candidemia onset) as risk factors for late mortality (30 days after candidemia onset).

Although ours is the largest study published to date regarding variables associated with septic shock in candidemic patients, it has some limitations that should be addressed. We only examine patients who develop septic shock within the first $24 \mathrm{~h}$ from the first positive blood culture for Candida. Therefore, we cannot rule out that some patients presented with a septic shock in a later 
stage of the infection. Furthermore, we did not include, in the risk factors analysis for mortality, other core elements of general supportive care that are crucial for an adequate septic shock management (need for mechanical ventilation, use of intravenous fluids and oxygen therapy). Therefore, additional studies specifically designed to investigate risk factors predicting septic shock development in patients with candidemia may be useful to assess the reproducibility of our results.

\section{Conclusions}

An abdominal source of the infection is the most important factor significantly associated with the development of septic shock attributable to candidemia. In view of the prognostic implication of septic shock development, our results lead us to consider an appropriate stratification of candidemic patients on the basis of the source of the infection.

\section{Supplementary information}

Supplementary information accompanies this paper at https://doi.org/10. 1186/s13054-020-2793-y.

Additional file 1. Comparison between patients with and without intraabdominal origin of candidemia.

\section{Abbreviations}

CVC: Central venous catheter; ICU: Intensive care unit; OR: Odds ratio

\section{Acknowledgements}

Not applicable

\section{Endorsement}

The study was endorsed by the Critically III Patients Study Group (ESGCIP) of the European Society of Clinical Microbiology and Infectious Diseases (ESCMID), by the Grupo de Estudio de Micología Médica (GEMICOMED) dentro de la SEIMC (Sociedad Española de Enfermedades infecciosas y Microbiología Clinica) and by Società Italiana di Terapia Antinfettiva (SITA).

\section{Authors' contributions}

$\mathrm{MB}, \mathrm{PM}, \mathrm{CGV}, \mathrm{EB}, \mathrm{MM}$, and $\mathrm{AV}$ made substantial contributions to the study concept and design, acquisition of data, analysis and interpretation of data, first drafting of the manuscript, and critical revision of the manuscript for important intellectual content. All the other authors made substantial contributions to the acquisition of data and critical revision of the manuscript for important intellectual content. All authors read and approved the final manuscript.

\section{Funding}

This study was funded by a research grant from the Ministerio de Sanidad y Consumo, Instituto de Salud Carlos III [FIS PI15/00744], European Regional Development Fund (ERDF); CGV is a recipient of an INTENSIFICACIÓ Grant from the "Strategic plan for research and innovation in health-PERIS 2016$2020^{\prime \prime}$ and forms part of the Fungi CLINIC Research group (AGAUR-Project 2017SGR1432 of the Catalan Health Agency).

\section{Availability of data and materials}

The datasets used and/or analyzed during the current study are available from the corresponding author on reasonable request.

\section{Ethics approval and consent to participate}

The study was approved by the local institutional review boards, and written patient consent was not required because of observational nature of this study.

\section{Consent for publication}

Not applicable

\section{Competing interests}

MB has participated in advisory boards and/or received speaker honoraria from Achaogen, Angelini, Astellas, AstraZeneca, Bayer, Basilea, Cidara, Gilead, Menarini, MSD, Paratek, Pfizer, Roche, The Medicine Company, Shionogi, Tetraphase, VenatoRx, and Vifor. CGV has received honoraria for talks on behalf of Gilead Science, Merck Sharp and Dohme, Novartis, Pfizer, Jannsen, as well as a grant from Gilead Science. JC has received honoraria for lectures from Gilead and Merck Sharp and Dohme. MS has received honoraria for participating in meetings, forums and consultancies, as well as for having given conferences for MSD, Pfizer, Janssen, Angelini, ERN, Gilead, and Astellas $\mathrm{Ph}$. He has also received grants and scholarships for research and teaching, which were administered through La Fe Sanitary Research Institute (IIS-LA FE) and companies such as ViiV, Janssen, MSD. LELC has served as speaker for Merck, Sharp, and Dohme. Angelini has received research support from Novartis and served as trainer for Merck, Sharp, and Dohme. PM is a consultant and/or speaker for Astellas, Gilead, Merck, Novartis, and Pfizer, and T2 Biosystems. JF has been advisor/consultant and has received honoraria for talks on behalf of Gilead Sciences, Merck, Sharp, and Dohme, Pfizer, and Astellas Pharma; MFR has received honoraria for talks on behalf of Gilead Science, Astellas, and Pfizer. JMA has received honoraria for speaking at symposia organized on behalf of Pfizer, Astellas, Merck, Sharp, \& Dohme (MSD). Angelini has sat on advisory boards for antifungal agents on behalf of Pfizer, Astellas, MSD, Angelini, and Gilead Science.

\section{Author details}

${ }^{1}$ Infectious Diseases Clinic, Department of Medicine, University of Udine and Azienda Sanitaria Universitaria Integrata, Piazzale Santa Maria della Misericordia 15, 33010 Udine, Italy. ${ }^{2}$ Department of Health Sciences, University of Genoa, Genoa, Italy. ${ }^{3}$ Clinica Malattie Infettive, Ospedale Policlinico San Martino-IRCCS, Genoa, Italy. ${ }^{4}$ Hospital Clínic, IDIBAPS (Institut d'Investigacions biomèdiques Agust Pi i Sunyer), Universitat de Barcelona, Barcelona, Spain. ${ }^{5}$ Hospital Universitari de Bellvitge, IDIBELL (Institut D'Investigació Biomèdica de Bellvitge), Universitat de Barcelona, Barcelona, Spain. ${ }^{6}$ Hospital Universitari I Politecnic "La Fe", Valencia, Spain. ${ }^{7}$ Hospital Universitario Clínico "San Carlos", Madrid, Spain. ${ }^{8}$ Hospital Universitario "Ramón y Cajal", Madrid, Spain. 'Hospital Universitario "12 de Octubre", Instituto de Investigación Hospital "12 de Octubre" (i+12), Universidad Complutense de Madrid, Madrid, Spain. ${ }^{10}$ Unidad Clínica de Enfermedades Infecciosas y Microbiología Clínica, Hospital Universitario Virgen Macarena/ Instituto de Biomedicina de Sevilla (IBiS)/Universidad de Sevilla/Centro Superior de Investigaciones Científicas, Seville, Spain. ${ }^{11}$ Hospital Universitari Vall d'Hebron, VHIR (Vall d'Hebron Institut de Recerca), Universitat Autònoma de Barcelona, Barcelona, Spain. ${ }^{12}$ Hospital Universitario "Cruces", Bilbao, Spain. ${ }^{13}$ Hospital Universitario "Virgen del Rocío", Sevilla, Spain. ${ }^{14}$ Clinical Microbiology and Infectious Diseases, Hospital General Universitario Gregorio Marañón, Madrid, Spain. ${ }^{15}$ Instituto de Investigación Sanitaria, Hospital Gregorio Marañón, Madrid, Spain. ${ }^{16}$ Microbiology Unit, Azienda Sanitaria Universitaria Integrata Santa Maria della Misericordia, Udine, Italy. ${ }^{17}$ Medicine Department School of Medicine, Universidad Complutense de Madrid, Madrid, Spain. ${ }^{18}$ CIBER Enfermedades Respiratorias-CIBERES (CB06/06/0058), Udine, Spain.

Received: 19 October 2019 Accepted: 17 February 2020

Published online: 26 March 2020

\section{References}

1. Martin GS, Mannino DM, Eaton S, Moss M. The epidemiology of sepsis in the United States from 1979 through 2000. N Engl J Med. 2003;348(16): 1546-54.

2. Payen DM, Guilhot J, Launey Y, Lukaszewicz AC, Kaaki M, Veber B, et al. Early use of polymyxin B hemoperfusion in patients with septic shock due to peritonitis: a multicenter randomized control trial. Intensive Care Med. 2015; 41(6):975-84.

3. Bassetti M, Vena A, Russo A. Management of patients with septic shock due to Candida infection. Hosp Pract (1995). 2018;46(5):258-65.

4. Bassetti M, Righi E, Ansaldi F, Merelli M, Trucchi C, De Pascale G, et al. A multicenter study of septic shock due to candidemia: outcomes and predictors of mortality. Intensive Care Med. 2014;40(6):839-45.

5. Kollef M, Micek S, Hampton N, Doherty JA, Kumar A. Septic shock attributed to Candida infection: importance of empiric therapy and source control. Clin Infect Dis. 2012;54(12):1739-46. 
6. Bassetti M, Giacobbe DR, Vena A, Trucchi C, Ansaldi F, Antonelli M, et al. Incidence and outcome of invasive candidiasis in intensive care units (ICUs) in Europe: results of the EUCANDICU project. Crit Care. 2019;23(1):219.

7. Guzman JA, Tchokonte R, Sobel JD. Septic shock due to candidemia: outcomes and predictors of shock development. J Clin Med Res. 2011;3(2):65-71.

8. Charlson ME, Pompei P, Ales KL, MacKenzie CR. A new method of classifying prognostic comorbidity in longitudinal studies: development and validation. J Chronic Dis. 1987;40(5):373-83.

9. Singer M, Deutschman CS, Seymour CW, Shankar-Hari M, Annane D, Bauer $M$, et al. The Third International Consensus Definitions for Sepsis and Septic Shock (Sepsis-3). JAMA. 2016;315(8):801-10.

10. Pappas PG, Kauffman CA, Andes DR, Clancy CJ, Marr KA, Ostrosky-Zeichner L, et al. Clinical Practice Guideline for the Management of Candidiasis: 2016 Update by the Infectious Diseases Society of America. Clin Infect Dis. 2016; 62(4):e1-50.

11. Mermel LA, Allon M, Bouza E, Craven DE, Flynn P, O'Grady NP, et al. Clinical practice guidelines for the diagnosis and management of intravascular catheter-related infection: 2009 Update by the Infectious Diseases Society of America. Clin Infect Dis. 2009:49(1):1-45.

12. Cuervo G, Garcia-Vidal C, Puig-Asensio M, Vena A, Meije Y, Fernandez-Ruiz M, et al. Echinocandins compared to fluconazole for candidemia of a urinary tract source: a propensity score analysis. Clin Infect Dis. 2017;64(10):1374-9.

13. Bassetti M, Peghin M, Carnelutti A, Righi E, Merelli M, Ansaldi F, et al. Clinical characteristics and predictors of mortality in cirrhotic patients with candidemia and intra-abdominal candidiasis: a multicenter study. Intensive Care Med. 2017;43(4):509-18.

14. Bassetti M, Righi E, Ansaldi F, Merelli M, Scarparo C, Antonelli M, et al. A multicenter multinational study of abdominal candidiasis: epidemiology, outcomes and predictors of mortality. Intensive Care Med. 2015:41(9):1601-10.

15. Bassetti M, Marchetti M, Chakrabarti A, Colizza S, Garnacho-Montero J, Kett $\mathrm{DH}$, et al. A research agenda on the management of intra-abdominal candidiasis: results from a consensus of multinational experts. Intensive Care Med. 2013;39(12):2092-106

16. Agnelli C, Valerio M, Bouza E, Vena A, Guinea J, Del Carmen M-JM, et al. Persistent Candidemia in adults: underlying causes and clinical significance in the antifungal stewardship era. Eur J Clin Microbiol Infect Dis. 2019;38(3): 607-14.

17. Cuervo G, Garcia-Vidal C, Puig-Asensio M, Merino P, Vena A, Martin-Pena A et al. Usefulness of guideline recommendations for prognosis in patients with candidemia. Med Mycol. 2019;57(6):659-67.

18. Sbrana F, Sozio E, Bassetti M, Ripoli A, Pieralli F, Azzini AM, et al. Independent risk factors for mortality in critically ill patients with candidemia on Italian Internal Medicine Wards. Intern Emerg Med. 2018;13(2):199-204.

19. Patel GP, Simon D, Scheetz M, Crank CW, Lodise T, Patel N. The effect of time to antifungal therapy on mortality in Candidemia associated septic shock. Am J Ther. 2009:16(6):508-11.

20. Ng K, Schorr C, Reboli AC, Zanotti S, Tsigrelis C. Incidence and mortality of sepsis, severe sepsis, and septic shock in intensive care unit patients with candidemia. Infect Dis (Lond). 2015:47(8):584-7.

21. Haydar S, Spanier M, Weems P, Wood S, Strout T. Comparison of QSOFA score and SIRS criteria as screening mechanisms for emergency department sepsis. Am J Emerg Med. 2017;35(11):1730-3.

22. Askim A, Moser F, Gustad LT, Stene H, Gundersen M, Asvold BO, et al. Poor performance of quick-SOFA (qSOFA) score in predicting severe sepsis and mortality - a prospective study of patients admitted with infection to the emergency department. Scand J Trauma Resusc Emerg Med. 2017;25(1):56.

23. Churpek MM, Snyder A, Sokol S, Pettit NN, Edelson DP. Investigating the impact of different suspicion of infection criteria on the accuracy of quick sepsis-related organ failure assessment, systemic inflammatory response syndrome, and early warning scores. Crit Care Med. 2017:45(11):1805-12

24. Bassetti M, Molinari MP, Mussap M, Viscoli C, Righi E. Candidaemia in internal medicine departments: the burden of a rising problem. Clin Microbiol Infect. 2013;19(6):E281-4.

25. Vena A, Bouza E, Valerio M, Padilla B, Pano-Pardo JR, Fernandez-Ruiz M, et al. Candidemia in non-ICU surgical wards: comparison with medical wards. PLoS One. 2017;12(10):e0185339.

26. Tascini C, Falcone M, Bassetti M, De Rosa FG, Sozio E, Russo A, et al. Candidemia in patients with body temperature below 37 degrees $C$ and admitted to internal medicine wards: assessment of risk factors. Am J Med. 2016;129(12):1330 e1-6.
27. Luzzati R, Merelli M, Ansaldi F, Rosin C, Azzini A, Cavinato S, et al. Nosocomial candidemia in patients admitted to medicine wards compared to other wards: a multicentre study. Infection. 2016;44(6):747-55.

28. Tedeschi S, Tumietto F, Giannella M, Bartoletti M, Cristini F, Cioni G, et al. Epidemiology and outcome of candidemia in internal medicine wards: a regional study in Italy. Eur J Intern Med. 2016;34:39-44.

29. Panpetch W, Somboonna N, Bulan DE, Issara-Amphorn J, Worasilchai N, Finkelman $\mathrm{M}$, et al. Gastrointestinal colonization of Candida albicans increases serum (1-->3)-beta-D-glucan, without candidemia, and worsens cecal ligation and puncture sepsis in murine model. Shock. 2018;49(1):6270.

30. Hiengrach P, Panpetch W, Worasilchai N, Chindamporn A, Tumwasorn S, Jaroonwitchawan T, et al. Administration of Candida albicans to dextran sulfate solution treated mice causes intestinal dysbiosis, emergence and dissemination of intestinal Pseudomonas aeruginosa and lethal sepsis. Shock. 2020;53(2):189-98.

31. Panpetch W, Somboonna N, Bulan DE, Issara-Amphorn J, Finkelman M, Worasilchai $\mathrm{N}$, et al. Oral administration of live- or heat-killed Candida albicans worsened cecal ligation and puncture sepsis in a murine model possibly due to an increased serum (1-->3)-beta-D-glucan. PLoS One. 2017; 12(7):e0181439.

32. Tissot F, Lamoth F, Hauser PM, Orasch C, Fluckiger U, Siegemund M, et al. Beta-glucan antigenemia anticipates diagnosis of blood culture-negative intraabdominal candidiasis. Am J Respir Crit Care Med. 2013;188(9):1100-9.

33. Martin-Mazuelos E, Loza A, Castro C, Macias D, Zakariya I, Saavedra P, et al. Beta-D-Glucan and Candida albicans germ tube antibody in ICU patients with invasive candidiasis. Intensive Care Med. 2015;41(8):1424-32.

34. Munoz P, Vena A, Machado M, Gioia F, Martinez-Jimenez MC, Gomez E, et al. T2Candida MR as a predictor of outcome in patients with suspected invasive candidiasis starting empirical antifungal treatment: a prospective pilot study. J Antimicrob Chemother. 2018;73(suppl_4):iv6-iv12.

35. Fortun J, Gioia F, Munoz P, Graus J, Gomez-Garcia de la Pedrosa E, MartinDavila $P$, et al. T2 magnetic resonance for the diagnosis of deep-seated invasive candidiasis in a liver recipient without candidemia. Rev Iberoam Micol 2018;35(3):159-161.

36. Rosenblatt R, Mehta A, Cohen-Mekelburg S, Shen N, Snell D, Lucero C, et al. The rise of Clostridioides difficile infections and fall of associated mortality in hospitalized advanced cirrhotics. Liver Int. 2019;39(7):1263-70.

37. Larkin E, Hager C, Chandra J, Mukherjee PK, Retuerto M, Salem I, et al. The emerging pathogen Candida auris: growth phenotype, virulence factors, activity of antifungals, and effect of SCY-078, a novel glucan synthesis inhibitor, on growth morphology and biofilm formation. Antimicrob Agents Chemother. 2017;61(5).

38. Machado FR, Ferreira EM, Schippers P, de Paula IC, Saes LSV, de Oliveira FI $\mathrm{Jr}$, et al. Implementation of sepsis bundles in public hospitals in Brazil: a prospective study with heterogeneous results. Crit Care. 2017;21(1):268.

39. Lynn NB, Gupta C, Vaaler M, Held J, Leon L. Severe sepsis 3-hour bundle compliance and mortality. Am J Infect Control. 2018:46(11):1299-300.

40. Milano PK, Desai SA, Eiting EA, Hofmann EF, Lam CN, Menchine M. Sepsis bundle adherence is associated with improved survival in severe sepsis or septic shock. West J Emerg Med. 2018;19(5):774-81.

41. Puig-Asensio M, Padilla B, Garnacho-Montero J, Zaragoza O, Aguado JM, Zaragoza $\mathrm{R}$, et al. Epidemiology and predictive factors for early and late mortality in Candida bloodstream infections: a population-based surveillance in Spain. Clin Microbiol Infect. 2014:20(4):0245-54.

\section{Publisher's Note}

Springer Nature remains neutral with regard to jurisdictional claims in published maps and institutional affiliations. 